\title{
Die Tücken der Kommunikation im Arztberuf Immer an Schweigepflicht und Datenschutz denken
}

\begin{abstract}
Nicht alles was im Rahmen der Kommunikation im Arztberuf möglich ist, ist auch erlaubt. Insbesondere die Schweigepflicht und der Datenschutz sind regelmäßig auch bei der Verwendung technischer Kommunikationswege - zu berücksichtigen. Informieren Sie sich also gründlich über die notwendigen Sicherheitsstandards, bevor Sie zum Beispiel Arztbriefe per E-Mail verschicken. Tücken bergen unter Umständen auch Aufklärungsgespräche. So ist der Arzt nicht nur für einen angemessenen zeitlichen Abstand zu dem geplanten Eingriff verantwortlich, sondern auch für eine verständliche Formulierung der Inhalte, was insbesondere bei nicht deutschsprachigen Patienten immer wieder problematisch sein kann.
\end{abstract}

Kommunikation unter Ärzten und mit Patienten ist ein wesentlicher Teil der medizinischen Behandlung. Sie findet auf diversen Ebenen statt und kann die unterschiedlichsten Probleme juristischer Art aufwerfen - sei es bei Arztbriefen, Aufklärungsgesprächen oder auch im Rahmen von telemedizinischen Maßnahmen.

Genau wie bei der Behandlung selbst sind also auch bei der Kommunikation zwischen Ärzten untereinander und zwischen Ärzten und Patienten viele juristische Spielregeln zu beachten. Zahlreiche Fallstricke hält zum Beispiel die Kommunikation der Ärzte eines Krankenhauses mit Patienten bereit. Kernpunkte sind dabei die ärztliche Schweigepflicht und die Aufklärung der Patienten, die mehr Probleme bergen können als es auf den ersten Blick scheint.

\section{Aufklärungsfristen einhalten}

Bezüglich der Aufklärung der Patienten gilt zunächst grundsätzlich eines: Spätestens einen Tag vor einem Eingriff, so die gängige Rechtsprechung, sollte der Operateur mit dem Patienten über die möglichen Risiken des geplanten Eingriffs sprechen. In bestimmten Fällen sogenannten „Notfalloperationen“ kann aber auch ein deutlich kürzerer Zeitraum zwischen dem Aufklärungsgespräch und dem Eingriff erlaubt sein, dann nämlich, wenn durch eine Verzögerung des Eingriffs auf den nachfolgenden Tag gewichtige Komplikationen zu befürchten sind oder sich die Hei- lungsraten deutlich verschlechtern. Konkret bedeutet dies: Je dringlicher der Eingriff ist, umso kürzer kann auch die „Bedenkzeit“ für den Patienten ausfallen (vgl. klinikarzt 2007; 36 (12): 676-677).

\section{Mögliches Vetorecht minder- jähriger Patienten}

Bei minderjährigen Patienten wiederum ist zu beachten, dass - auch wenn die Aufklärung in diesem Fall grundsätzlich gegenüber den Eltern erfolgen muss - den Jugendlichen unter Umständen ebenfalls ein Vetorecht zugebilligt werden muss. Einen Eingriff ablehnen kann der Minderjährige, wenn er über eine ausreichende Urteilsfähigkeit verfügt (hier ist das Urteilsvermögen des Arztes gefragt), der geplante Eingriff nur relativ indiziert ist und möglicherweise erhebliche Folgen für die künftige Lebensgestaltung des Minderjährigen auftreten können (vgl. klinikarzt 2007; 36 (2): 69-70).

\section{Verständigungsprobleme mit fremdsprachigen Patienten}

Besonders problematisch sind Aufklärungsgespräche jedoch, wenn der Patient der deutschen Sprache nicht mächtig ist. Grundsätzlich gilt in diesen Fällen der gleiche strenge Maßstab wie bei deutschen Patienten. Die ärztliche Behandlung setzt immer eine Einwilligung des Patienten voraus. Dazu wiederum muss der Patient das Wesen, die Bedeutung und die Tragweite der Maß- nahme kennen. Dieses Wissen erlangt der Patient durch die Aufklärung des Arztes. Der Arzt muss also vorab mit dem Patienten kommunizieren, um die richtige Diagnose stellen zu können und dem Patienten erläutern, welche Behandlung er bei ihm vornehmen möchte.

Bei einem nicht deutschsprachigen Patienten muss daher sichergestellt sein, dass dieser trotz eventueller Verständigungsprobleme ein ausreichendes Bild über die geplante Therapie und deren potenzielle Risiken erhält. Nur dann kann er wirksam in die Behandlung einwilligen. Wie sich der Arzt verständlich macht, bleibt ihm selbst überlassen. Nach der Rechtsprechung hat er allerdings die Pflicht, sich bei aufkommenden Sprach- und Verständigungsschwierigkeiten (Ausnahme Notfall) zumindest eines der Sprache des Patienten mächtigen Mitarbeiters, eines Familienangehörigen oder im Extremfall eines Dolmetschers zu bedienen.

Ein Extremfall wäre wohl dann anzunehmen, wenn der Arzt den Eindruck hat, dass der Patient trotz dieser Maßnahmen nicht versteht, was ihm vermittelt werden soll. Daher muss sich der Arzt davon überzeugen, dass die Sprachkenntnisse der herangezogenen Hilfsperson ausreichen, um den aufklärungsbedürftigen Sachverhalt hinreichend verständlich zu erläutern. Denn die Verantwortung dafür, dass der Patient alles verstanden hat, trägt der behandelnde Arzt.

Denken Sie auch daran: Immer wenn Sie Dritte zu einem Aufklärungsgespräch hinzuziehen, müssen Sie sich von der Schweigepflicht entbinden lassen - das gilt auch, wenn es sich um Angehörige des Patienten handelt.

\section{Schweigepflicht ist weit gefasst}

Gerade die ärztliche Schweigepflicht wird oft unterschätzt: Bereits vermeintlich unverfängliche Angaben, wie 
der Name und die Anschrift des Patienten oder allein die Tatsache, dass ein Patient in Behandlung ist, fallen unter die Schweigepflicht eines Arztes (vgl. z. B. klinikarzt 2007; 36 (10): 555-556). Daher sollte man grundsätzlich keinerlei Angaben über einen Patienten weitergeben, wenn man nicht im Vorfeld von diesem von der Schweigepflicht entbunden wurde. Das gilt übrigens auch dann, wenn ein Patient Auskunft über einen Mitpatienten verlangt, um gegen diesen Schadensersatzansprüche durchsetzen zu können. Natürlich spielt die ärztliche Schweigepflicht auch eine wesentliche Rolle bei Informationen gegenüber Angehörigen der Patienten.

\section{Geheimhaltungspflicht auch gegenüber Kollegen}

Als zentrale Berufspflicht eines Arztes schränkt die Schweigepflicht zudem die ärztliche Kommunikation erheblich ein. Sie gilt vom Grundsatz her uneingeschränkt gegenüber jedermann, der nicht in das konkrete Arzt-PatientenVerhältnis einbezogen ist. Die Geheimhaltungspflicht besteht demnach auch im Verhältnis von Ärzten untereinander.

Dass die Schweigepflicht aufgehoben sei, wenn der Empfänger der Mitteilung seinerseits schweigepflichtig ist, ist ein weit verbreiteter Irrglaube. Eine Offenbarungsbefugnis besteht nur insoweit, als der andere Arzt in das Behandlungsgeschehen eingebunden ist (etwa, wenn er konsiliarisch hinzugezogen wird) und deswegen das Einverständnis des Patienten zum Beispiel im Wege der mutmaßlichen Einwilligung anzunehmen ist.

\section{Eingriff des Gesetzgebers in die Kommunikation unter Ärzten}

Bisweilen greift sogar der Gesetzgeber in die Kommunikation von Ärzten untereinander ein. So wurde zum Beispiel im Fünften Sozialgesetzbuch zunächst vorgeschrieben, dass Krankenhausärzte in Entlassungsbriefen nur noch den Wirkstoff eines Arzneimittels aber nicht den Produktnamen nennen dürfen. Dies sollte dem niedergelassenen Arzt die Möglichkeit geben, zumindest ein günstigeres Produkt als das im Krankenhaus Verabreichte zu verordnen, ohne mit den Patienten in belastende Diskussionen einsteigen zu müssen.

Technischer Fortschritt setzt Grundregeln der ärztlichen Kommunikation nicht außer Kraft

Die fortschreitende Technisierung, angefangen bei Internet, E-Mail und Fax bis hin zu den neuesten technischen Möglichkeiten in Bezug auf die Telemedizin, erweitert die Kommunikationsmöglichkeiten der Ärzte beträchtlich. So wichtig und praktisch die nahezu unbeschränkte Austauschmöglichkeit von Informationen aber auch ist, so dürfen natürlich die Grenzen des Austausches - wie etwa die Schweigepflicht und der Datenschutz - dabei nicht in den Hintergrund treten. Diese grundlegenden Regeln bei der Kommunikation im Arztberuf behalten nicht nur weiterhin ihre Gültigkeit, sie gewinnen sogar noch an Bedeutung!

\section{Arztbrief/Entlassungsbericht als E-Mail?}

Der bis dato übliche Weg, Arztbriefe oder Entlassungsberichte zu versenden, ist nach wie vor der Postweg. Durch die stetige Technisierung stellt sich aber natürlich auch die Frage, ob solche vertraulichen Patientendaten auch per E-Mail verschickt werden dürfen. Die Musterberufsordnung der Ärzte enthält kein ausdrückliches Verbot, Arztbriefe oder Entlassungsberichte per E-Mail zu versenden. Jedoch müssen die Anforderungen an die Schweigepflicht mit größter Sorgfalt berücksichtigt werden, da das informationelle Selbstbestimmungsrecht des Patienten durch die moderne EDV-mäßige Datenverarbeitung am stärksten gefährdet wird.

Patienteninformationen sind persönliche Daten, daher gelten hier besonders hohe Ansprüche an Vertraulichkeit und Schutz vor Veränderungen. Da das Versenden von E-Mails auf einer Ebene mit dem Verschicken von Postkarten steht, müssen zusätzliche technische und organisatorische Maßnahmen vorliegen, um eine möglichst hohe Sicherheit bei dem Umgang mit diesen Daten zu erreichen. Wer Patientendaten über das Internet verschicken will, muss sich daher über alle datenschutzrechtlichen und juristischen Anforderungen im Vorfeld informieren und diese unbedingt einhalten.

\section{Datenschutz im Rahmen der Telemedizin}

Telemedizin ist die kombinierte Anwendung von Telekommunikation und Informatik (Telematik). Sie ermöglicht oder unterstützt, in Überwindung räumlicher Entfernungen, medizinische Dienstleistungen (wie etwa die Befundung von in München erstellten Röntgenbildern durch einen Arzt in Hamburg). Es fehlt daher ein unmittelbarer Arzt-Patienten-Kontakt.

Die Möglichkeiten des Austausches von Patientendaten im Bereich der Telemedizin zwischen behandelnden Ärzten oder mit Dritten ergeben für den Patienten allerdings neue Risiken und Gefährdungen des Rechts auf informationelle Selbstbestimmung. Aus Gründen des Datenschutzes und der Schweigepflicht sind daher auch hier besondere Maßnahmen erforderlich, um den im Rahmen telemedizinischer Anwendungen erfolgenden Datenfluss kontrollieren zu können. Dementsprechend sind bei der Telemedizin ebenfalls erhebliche juristische und datenschutzrechtliche Klippen zu nehmen, über die man sich in jedem Fall vorab informieren sollte.

Allerdings schlug diese Regelung mangels ausreichender Beachtung der Krankenhäuser fehl. Jetzt gilt: Soweit dies ohne eine Beeinträchtigung der Behandlung im Einzelfall oder ohne eine Verlängerung der Verweildauer möglich ist, sollen im Rahmen der stationären Versorgung eines Patienten vor dessen Entlassung Arzneimittel eingesetzt werden, die auch bei Verordnung in der vertragsärztlichen Versorgung zweckmäßig und wirtschaftlich sind (vgl. klinikarzt 2006; 35 (6): XVIII-XIX).

\section{Kommunikation mit}

\section{Krankenkassen und MDK}

Erhebliche Probleme können zudem im Rahmen der Kommunikation mit Krankenkassen und deren medizinischen Diensten (MDK) entstehen. Zentrales Problem ist hier in der Regel das Einsichtsbegehren der Kassen in die Patientenunterlagen, dem die ärztliche Schweigepflicht entgegen steht.

\section{Fazit}

Nicht alles was im Rahmen der Kommunikation im Arztberuf möglich ist, ist auch erlaubt. Insbesondere die Schweigepflicht und der Datenschutz sind zwei zentrale Hürden, die sich im klinischen Alltag häufiger stellen als gedacht. Im Zweifelsfall sollte man daher immer den Rat eines Juristen einholen.

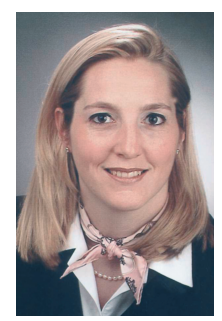

Korrespondenz Dr. iur. Isabel Häser Rechtsanwältin Ehlers, Ehlers \& Partner Widenmayerstraße 29 80538 München i.haeser@eep-law.de 